\title{
Research on Optimization Strategy of New Energy Grid Scheduling
}

\author{
Feihong $\mathrm{Wu}^{1,2}$, Gengyin $\mathrm{Li}^{1}$, Yao Wang ${ }^{3}$ and Chen $\mathrm{Zhao}^{2}$ \\ ${ }^{1}$ North China Electric Power University, Beijing 102206, China
${ }^{2}$ State Grid Shanxi Electric Power Company Maintenance Company, Xiaodian District 030032 , Taiyuan, China

${ }^{3}$ State Grid Shanxi Economic and Technical Research Institute, Yingze District 030002, Taiyuan, China

Keywords: new energy, grid-connected, scheduling system.

\begin{abstract}
In recent years, the development and construction of new energy in China has gradually increased. The generating capacity of solar energy, wind energy etc. new energy power generation has increased year by year. A large number of parallel operation of new energy become the power grid dispatching automation system to face a new challenge. Now mostly power grid dispatching automation system can only monitor the working status of new energy booster station and difficult to effectively control. With the increasing of new energy scale, its interconnection control has become an important problemin power system. Starting from the functions of dispatching automation system, some system control functions of the system were discussed and analyzed.
\end{abstract}

\section{Background}

With the rapid development of China's economy, energy consumption is increasing year by year, coal and oil is the most important traditional energy consumption in China, and the large consumption of traditional energy often cause great environmental damage, in addition, the traditional energy reserves are limited Of the new energy construction has become an important task of China's energy strategy. With the continuous development of technology, solar power and wind power has been rapid development, has been initially commercial model, a large number of wind and solar power generation equipment has been put into use. However, due to the volatility and irregularity of these energy sources, the new energy sources in the process of the existence of peaking, voltage regulation and thermal stability adjustment and many other issues, the smooth progress of the grid made a great challenge. In this paper, the structure of the new energy grid-connected dispatching support system is introduced, the basic functions of the system are discussed, and the main control functions of the system are studied.

\section{New Energy Grid-based Scheduling Support System of the Basic Functions}

New energy dispatching and grid support system is an important means for remote control center to perform new energy plant scheduling and monitoring. Dispatch system function is mainly to estimate the power of the plant site, the new energy and grid for automatic power generation control and automatic control of new energy sources. For the new energy support system software function structure, including production scheduling management system, real-time system, data support platform and WEB support system and network communication several parts. The real-time system mainly monitors and controls the production process data in real time. The data support platform mainly analyzes the real-time and historical data, and analyzes the data of the production process. Provides data support for power grid decision and operation status analysis. WEB support system provides customers with network sharing and management functions. Network communication provides communication support for remote data exchange. 


\section{Analysis on the Function of New Energy Grid - connected Dispatching Support System}

\subsection{New Energy Grid-connected Scheduling Support System Power Estimation System}

\section{(1) Power Estimation Analysis Model}

In the weather forecast data, select the required input data. If the research object is wind power, the fan height data should also be considered. The output variable should be used as the historical power data of the forecasting system. Due to the outer ring.

The change of the environment has a great influence on the power output of the new energy source, which leads to the fluctuation of the numerical value. In order to improve the accuracy of the BP neural network prediction data, the wavelet analysis of the data should be carried out. Because of the units and orders of magnitude of each input variable data in other cases, the data should be normalized before the analysis is performed. The steps of wavelet-neural network prediction are:

1) To maximize the accuracy of the estimated data, the application of wavelet analysis of data processing, and the implementation of normalized data processing;

2) The system automatically gives the network weights and network thresholds, determines the training function model and the number of times required for the network operation and determines the data such as learning efficiency;

3) The samples are divided into two parts: neural network training and verification, the samples used in the training network are sequentially input, and the backflush and output errors of each layer are calculated and the thresholds and weights are corrected. If $\mathrm{p}<\mathrm{P}$ Repeat this step;

4) To obtain the total error of the network, and analyze whether the training termination conditions, if not satisfied, then jump to step two;

5) Perform power estimates with the resulting network and perform error analysis on the results.

(2) The Structure of the Power Prediction System

The application of the new energy power prediction system structure layered structure in the new energy power generation forecasting system is beneficial to the field operation and the dispatch management. The factors that affect the estimated results are comprehensively considered, which is conducive to the optimization of the system, so as to improve the accuracy of the forecast results. The factors that need to be taken into account are weather forecast data, real-time meteorological data of regional area, maintenance plan of power station, and historical data of network operation. The data can be improved and the accuracy of prediction results can be improved.

\subsection{New Energy Grid Automatic Power Generation Control System}

\section{(1) The Function of the new Energy Automatic Power Generation Control System}

The new energy automatic power generation control system master station uses the network communication to send the control command to the monitoring system, the system carries on the automatic power generation control procedure as follows: The new energy dispatch main station uses the network communication to automatically generate electricity the control command is sent to the factory station. The station monitoring system determines whether the control command is executed according to the actual running status of the station and sets the state of the power generation control according to the execution status or the execution of the abandonment. When the command execution status is detected by the master station, the expected power value is sent to the new energy plant station, the automatic power generation control command is executed. When you need to exit the automatic power generation state, the master usually instructs the station to issue a command to exit the automatic power generation state. After receiving the exit command, the station exits the automatic power generation state and modifies the power generation status indication signal. New energy automatic power generation control system functions are:

1) to receive the power control command of the control center, and according to the command data to the site within the power distribution task; 2) the control system should be able to local and remote control two functions; 3) the system can be open-loop control and closed-loop control Control mode; 4) the system in line with the relevant requirements of safe and secure; 5) in order to improve the service life of equipment, equipment, the number of adjustments should be as little as possible. 


\section{(2) The Structure of New Energy Automatic Power Generation Control System}

The structure of the new energy automatic power generation control system is usually composed of maintenance work station and intelligent communication management equipment. The core of the entire system is intelligent communication equipment, which is mainly responsible for the various parts of the system data exchange, with information collection, data upload and automatic power generation control and other functions. Maintain the main function of the workstation for the monitoring and maintenance system, which through the network and intelligent communication management equipment connected. Intelligent communication management equipment using the network and monitoring system for data exchange, collection of wind turbines or solar photovoltaic power generation operation of real-time data, such as power supply power output, power supply voltage and power supply current and other data; and remote system data exchange is the power information Upload, and send the power generation automatic control action command to the execution system at the same time. Intelligent communication management equipment can be based on a given power command, a comprehensive analysis of the power grid and power generation equipment, the specific situation, by using the relevant algorithm to obtain the value of active power, so active power control.

\subsection{New Energy Grid Automatic Voltage Control System}

\section{(1) Automatic Voltage Control System Structure}

New energy and grid automatic voltage control system structure Intelligent communication equipment will be the new energy power generation equipment, reactive power compensation equipment, the operation of data transmission to the control center, while the voltage regulation command transmission to these devices. By analyzing the station equipment operating status and voltage regulation target, calculate the transformer joint position and reactive power compensation target value, and adjust the control instructions to the control device. In order to save resources, automatic power generation control and automatic voltage control can be integrated in a system.

(2) Automatic Power Generation Control and Automatic Voltage Control Program Design

Control the operation of the program, the program running the specific process:

1) Receives the control target value issued by the parent control center and reads the system measurement data. The measurement data mainly includes remote measurement data, remote communication transmission data and system protection parameters. The remote communication data are the value of the circuit breaker, grounding switch, line switch and disconnector. The system protection parameters mainly include transformer protection, line protection, capacitance reactance Protection and SVG and SVC protection signals.

2) Check whether the control system to achieve the adjustment target, that is, check whether the system adjustment results meet the convergence conditions.

3) Check the system to adjust the restrictions, control equipment, the minimum adjustment interval and the maximum adjustment frequency.

4) The system of measured values and equipment status detection, analysis of the system to meet the locking conditions, when the system failure occurs, the opening switch action to lock the system to prevent the accident to expand.

5) Calculate the automatic power generation control and automatic voltage control adjustment value, combined with the actual situation of the scene, the system active and reactive power distribution strategy.

6) Using the communication network of the substation automation control system, the control command is sent to the transformer tap, wind turbine, solar power generation equipment and SVC / SVG and so on to realize the adjustment and control of the relevant parameters.

\section{Conclusion}

In recent years, wind power and solar photo voltaic power generation and other new energy projects in full swing, get the country's strong support to strengthen the new energy grid scheduling support system development and construction of the power system to become a key project. In this 
paper, the functional structure of the new energy grid-connected dispatching support system is introduced and analyzed, and the system of power estimation, power generation control and voltage control are introduced. However, the scheduling support system there are many problems to be solved, such as energy storage, power prediction accuracy, etc., which requires the power system staff in practice continue to explore.

\section{References}

[1] Chai C, Lee W J, Fuangfoo P, et al. System impact study for the interconnection of wind generation and utility system. IEEE Trans on Industry Applications, 2005, Vol. 41(1)p. 163-168.

[2] Tu Qiang, Yi Lidong, Shang Yong, et al. Research on the application of power grid based synthetical regional wind power measurement system. Advances of Power System \& Hydroelectric Engineering, 2008, Vol. 24(1)p. 3-7.

[3] Xing Wenqi, Chao Qin, et al. Simulation study on wind power system containing various wind turbine generators.Power System Technology, 2009, Vol. 33(7)p. 103-106.

[4] Geng Hua, Xu Dewei, Wu Bin, et al. Control and stability analysis for the permanent magnetic synchronous generator based direct driven variable speed wind energy conversion system. Proceedings of the CSEE, 2009, Vol. 29(33)p. 68-75.

[5] Yi Lidong, Zhu Minyi, Wei Lei, et al. A computing method for peak load regulation ability of northwest China power grid connected with large-scale wind farms. Power System Technology, 2010, Vol. 34(2)p. 129-132.

[6] Sun Baogong, Ye Peng, Shao Guanghui, et al. Wind power penetration limit assessment based on nonlinear interior point algorithm. Proceedings of the CSEE, 2010, Vol. 30(10)p. 23-28.

[7] Fan Gaofeng, Zhao Haixiang, Dai Huizhu, et al. The impact and countermeasure of large scale wind power on power system. Power System and Clean Energy, 2008, Vol. 24(1)p. 44-48.

[8] Wang Xiaolan, Wang Mingwei, et al. Short-term wind speed forecasting based on wavelet decomposition and least square support vector machine. Power System Technology, 2010, Vol. 34(1)p. 179-184.

[9] Yuan Tiejiang, Chao Qin, Li Yiyan, et al. Short-term wind power output forecasting model for economic dispatch of power system incorporating large-scale wind farm6. Proceedings of the CSEE, 2010, Vol. 30(13)p. 23-27. 